\title{
Análise de sobrevivência aplicada ao desenvolvimento do pulgão Aphis gossypii Glover, 1877 (Hemiptera: Aphididae) em plantas de pepino (Cucumis sativus L.) contaminadas com fungicidas
}

\section{Survival analysis applied to the development of Aphis gossypii Glover, 1877 (Hemiptera: Aphididae) on fungicide-contaminated cucumber plants (Cucumis sativus $\mathbf{L}$.)}

\author{
Ronelza Rodrigues da Costa Zaché ${ }^{*}$; Geraldo Andrade Carvalho²; Marcelo \\ Ângelo Cirillo ${ }^{3}$; César Freire Carvalho ${ }^{2}$; Bruno Zaché ${ }^{1}$
}

\begin{abstract}
Resumo
Objetivou-se avaliar a influência de fungicidas utilizados em cultura de pepino sobre o desenvolvimento do pulgão Aphis gossypii Glover em condições de casa-de-vegetação. O delineamento experimental foi inteiramente ao acaso com quatro tratamentos e 45 repetições, sendo cada parcela composta por uma ninfa de primeiro ínstar, proveniente de uma fêmea áptera no início do período reprodutivo. Os fungicidas utilizados e suas respectivas dosagens em g i.a. $\mathrm{L}^{-1}$, foram enxofre $800 \mathrm{PM}(1,6)$, mancozebe 800 PM $(1,6)$ e oxicloreto de cobre 840 PM $(1,5)$. A testemunha foi composta somente por água. Os produtos avaliados não influenciaram o tempo mediano de vida em cada ínstar e da fase ninfal, sendo respectivamente de 1 e 4 dias. Oxicloreto de cobre e mancozebe aumentaram o tempo mediano de vida em dias do ciclo biológico total e da fase adulta desse afídeo.

Palavras-chave: Afídeo do algodoeiro, produto fitossanitário, cucurbitaceae, ambiente protegido
\end{abstract}

\begin{abstract}
It was aimed to evaluate the influence of fungicides utilized in cucumber on the development of the aphid Aphis gossypii Glover under greenhouse conditions. The experimental design was completely randomized with four treatments and 45 replicates, being each plot consisting of a first instar nymph coming from an apterous female in early reproductive period. The fungicides utilized and their respective dosages at g i.a.L $\mathrm{L}^{-1}$ were $800 \mathrm{PM}$ sulphur (1.6), $800 \mathrm{PM}$ mancozeb (1.6) and $840 \mathrm{PM}$ copper oxichloride (1.5). The control was made up of water only. The products did not influence the median time of life in each instar and the nymphal stage, being respectively 1 and 4 days. Copper oxychloride and mancozeb increased the median time of life in days and the total life cycle of the adult stage of this species.
\end{abstract}

Key words: Cotton aphid, pesticide, cucurbitaceae, protected environment

1 Doutorandos em Agronomia, Proteção de Plantas, Faculdade de Ciências Agronômicas de Botucatu/FCA-UNESP, Dept ${ }^{\circ}$ de Produção Vegetal, Cx. Postal 237, CEP 18610-307, Botucatu, SP. E-mail: ronelzagro@yahoo.com.br; bzache@bol.com.br

2 Prof. Dr. da Universidade Federal de Lavras, UFLA, Dept ${ }^{\circ}$ de Entomologia, DEN, Cx. Postal 3037, CEP 37200-000, Lavras, MG. E-mail: gacarval@ufla.br; cfcarvalho@ufla.br

3 Prof. Dr. da Universidade Federal de Lavras, UFLA, Dept ${ }^{0}$ de Estatística e Experimentação Agropecuária, Cx. Postal 3037, CEP 37200-000, Lavras MG. E-mail: marcelocirillo@hotmail.com

*Autor para correspondência 


\section{Introdução}

O pulgão Aphis gossypii Glover é uma das mais importantes pragas da cultura do pepino, sendo polífaga e apresenta ampla distribuição mundial (KOCOUREK et al., 1994). Além das cucurbitáceas, tem como plantas hospedeiras algodão, citros, café, cacau, berinjela, pimentão, batata, alface, cebola, brássicas e soja, além de muitas espécies ornamentais (BLACKMAN; EASTOP, 1984).

Esses insetos aglomeram-se, preferencialmente, na face inferior das folhas e nos brotos novos da planta hospedeira, causando danos diretos pela sucção de seiva ocasionando o encarquilhamento das folhas e a deformação dos brotos, e danos indiretos, pelo aparecimento da fumagina, com o desenvolvimento do fungo Capnodium spp., devido à excreção de honeydew, prejudicando a fotossíntese (DEGRANDE, 1998; MATTHEWS; TUNSTALL, 1994). Outro dano indireto é a transmissão de vírus (BARBOSA; FRANÇA, 1982; LEITE et al., 2008), sendo o mais importante vetor do vírus do mosaicodo-pepino (CMV) (ÁVILA, 1982).

A cultura de pepino cultivada em casa-devegetação pode favorecer o aparecimento de afídeos, os quais encontram ambiente ideal para a reprodução e desenvolvimento (BUENO, 2001), ou seja, as condições climáticas somadas à ausência de inimigos naturais, permitem que populações de $A$. gossypii aumentem a uma proporção acima de 0,5 fềmea/fêmea/dia (VANSTEENIS; EL-KHAWASS, 1995), o que equivale a um crescimento populacional de 3,5 vezes por semana.

As doenças, principalmente as fúngicas, também ocorrem com frequência nessa cultura, principalmente em ambiente protegido, sendo necessário o controle químico (JULIATTI, 2001).

Poucos são os estudos visando avaliar os efeitos dos fungicidas enxofre, mancozebe e oxicloreto de cobre sobre espécies de pulgões. Os produtos à base de cobre e enxofre são aplicados com sucesso em cultura de macieira, que segundo Balazs et al.
(1997), contribuem para o decréscimo dos afídeos Dysaphis devecta Walk., D. plantaginea Pass. e Aphis pomi De Geer, que são importantes pragas dessa cultura. Akhtar e van Emden (1992, 1996), e Weber e Kranz (1994) avaliaram a influência de fungicidas aplicados em cultivares de trigo de inverno sobre insetos-praga dessa cultura e constataram que pirazofós, propiconazol e fempropimorfe permitiram aumento significativo das populações de afídeos, e que anilazine causou redução populacional dos mesmos.

Assim, devido à aplicação de fungicidas ser uma prática frequente pelos horticultores, somada aos escassos estudos dos efeitos desses produtos sobre insetos pragas, o presente trabalho teve como objetivo avaliar a influência dos fungicidas enxofre, mancozebe e oxicloreto de cobre, aplicados em plantas de pepino, sobre o desenvolvimento do pulgão $A$. gossypii em casa-de-vegetação.

\section{Material e Métodos}

Os trabalhos foram conduzidos em casa-devegetação, com o cultivo de pepino (Cucumis sativus L.), em que quatro sementes do cultivar Caipira foram semeadas em vasos de polietileno de 1 L contendo mistura de terra de barranco e esterco de curral (3:1), mais 6,3 g de NPK na formulação 4-14-8, os quais foram mantidos em casa-de-vegetação. As plantas receberam adubação nitrogenada com $1,25 \mathrm{~g}$ de sulfato de amônia a cada 15 dias, sendo empregadas para a criação do pulgão A. gossypii e para condução dos experimentos em casa-de-vegetação.

A criação desse afídeo teve início a partir de espécimes oriundas da criação de manutenção do Laboratório de Biologia de Insetos do Departamento de Entomologia da UFLA, as quais foram transferidas para 15 plantas de pepino com 20 dias de idade, provenientes de cultivo em casade-vegetação. As plantas contendo os pulgões foram mantidas em cinco gaiolas acrílicas de $2 \mathrm{~m}$ de comprimento, $1 \mathrm{~m}$ de largura e $0,9 \mathrm{~m}$ de altura, com 
aberturas circulares laterais, vedadas com tecido voil, para permitir manuseio e promover aeração, na proporção de três plantas por gaiola. À medida que essas plantas entravam em senescência foram substituídas por outras, permitindo o deslocamento dos pulgões e a continuidade da criação.

Biologia de A. gossypii em plantas de pepino, contaminadas após pulverização com fungicidas em casa-de-vegetação

Plantas de pepino, com 20 dias de idade, receberam os fungicidas, com os respectivos nomes comerciais, técnicos e dosagens em g i.a.L-1 Kumulus 800PM - enxofre (1,6); Manzate 800PM mancozebe $(1,6)$; Recop 840PM - oxicloreto de cobre $(1,5)$ e água (testemunha), por meio de pulverizador manual até o ponto de escorrimento da calda, com volume médio de $7,5 \mathrm{~mL}$ por planta, e foram secas em condições ambiente. Posteriormente, duas fêmeas ápteras, foram liberadas em gaiola cilíndrica (4 cm de diâmetro) de plástico transparente fixada a folha por grampos. Após 16 horas, os adultos foram retirados deixando-se apenas uma ninfa neonata de $A$. gossypii de primeiro ínstar por gaiola/ planta, e as observações foram realizadas até o final do ciclo biológico desse inseto. As plantas foram irrigadas duas vezes ao dia e mantidas em casa-devegetação, no mês de março, a uma temperatura média de $26,5^{\circ} \mathrm{C}$ (mínima de $20^{\circ} \mathrm{C}$ e máxima de $33^{\circ} \mathrm{C}$ ) e umidade relativa média de $70 \%$, sendo distribuídas em delineamento inteiramente ao acaso, com quatro tratamentos (três fungicidas e a testemunha) com 45 repetições, sendo a unidade experimental formada por uma gaiola contendo um pulgão A. gossypii. Desta forma, a cada período de 24 horas as observações foram realizadas e o evento de interesse foi o registro de número de insetos mortos antes de completarem o tempo préestabelecido, caracterizando os dados censurados classificados por tipo I (COLOSIMO; GIOLO, 2005). Mediante ao exposto, convém ressaltar que as técnicas usuais de estatística experimental não foram consideradas na análise dos dados, uma vez que, os dados apresentaram um mecanismo de censura, uma vez que o estudo era encerrado após ter ocorrido o evento de interesse em um número pré-estabelecido de tempo, caracterizando a censura tipo I. Portanto, neste contexto, optou-se pelo uso do estimador não-paramétrico, do qual, foram geradas as curvas de sobrevivência considerando as estimativas da probabilidade de sobrevida até cada tempo de observação, independente da sobrevivência até outros períodos de observação. A interpretação dessas curvas foi realizada com o objetivo de comparar a sobrevivência de A. gossypii durante a fase ninfal, fase adulta (longevidade) e o ciclo biológico.

Em consonância com as curvas de sobrevivência estimadas, estimou-se o tempo mediano de vida (T), sendo aquele em que pelo menos $50 \%$ dos indivíduos de uma amostra se aproximaram do evento de interesse especificado (COLOSIMO; GIOLO, 2005).

Convencionou-se, nesse estudo, utilizar a probabilidade de sobrevivência (S) verificada no tempo mediano, sendo essa probabilidade considerada como a porcentagem de ninfas que ainda não sofreram ecdise no tempo mediano. Utilizou-se o procedimento LIFETEST do programa estatístico SAS (SAS, 1990) para obtenção das curvas de sobrevivência. Durante a fase ninfal o evento de interesse foi considerado a mudança de instar do pulgão e na fase adulta, o evento de interesse foi a morte do indivíduo, uma vez que a longevidade foi o parâmetro biológico avaliado.

\section{Resultados e Discussão}

Tempo de vida e probabilidade de sobrevivência

\section{/ Desenvolvimento e ciclo biológico}

Em todos os tratamentos, as ninfas de primeiro, segundo, terceiro e quarto ínstares de $A$. gossypii, criadas em plantas de pepino apresentaram um tempo mediano de vida de um dia (Tabela 1). Esses 
resultados assemelham-se aos obtidos por Vansteenis e El-Khawass (1995) para A. gossypii em plantas de pepino com 1,$0 ; 1,0$ e 0,7 dias para o primeiro, segundo e terceiro ínstares, respectivamente. E para o quarto ínstar, assemelhando-se aos verificados por Kocourek et al. (1994) com 1,2 dias.

Ninfas de primeiro e segundo instares em plantas dos tratamentos testemunha mancozebe, apresentaram probabilidade de sobrevivência de $10 \%$ (Tabela 1).

Para as ninfas de A. gossypii criadas em plantas de pepino no tratamento com enxofre, observou-se que a probabilidade de sobrevivência foi nula para todos os ínstares (Tabela 1). Quando as ninfas foram submetidas ao tratamento com oxicloreto de cobre, constatou-se que no tempo mediano de um dia, a probabilidade de sobrevivência foi de 26,$7 ; 16,0$; 20,0 e 20,0\%, para primeiro, segundo, terceiro e quarto ínstares (Tabela 1).

No terceiro e quarto ínstares, as ninfas da testemunha apresentaram no tempo mediano, probabilidade de sobrevivência de $20,0 \%$, sendo que $80,0 \%$ foi a porcentagem de ninfas de quarto ínstar e adultos, respectivamente, formadas nesse dia (Tabela 1). No tratamento com mancozebe, as ninfas de terceiro e quarto ínstares apresentaram o tempo mediano de vida de um dia e, nesse período, ainda havia 40,0 e $30,0 \%$ de ninfas para sofrerem ecdise (Tabela 1).

Tabela 1. Tempo mediano de vida (T) em dias e probabilidade de sobrevivência (S) em \% dos ínstares e da fase ninfal de Aphis gossypii mantidos em plantas de pepino tratados com fungicidas em casa-de-vegetação.

\begin{tabular}{lcccccccccccc}
\hline Tratamentos & \multicolumn{1}{c}{ Ínstares } & \multicolumn{1}{c}{ Fase ninfal } \\
\cline { 2 - 9 } & \multicolumn{1}{c}{ Primeiro } & \multicolumn{1}{c}{ Segundo } & Terceiro & \multicolumn{2}{c}{ Quarto } & \\
\cline { 2 - 8 } & $\mathbf{T}$ & $\mathbf{S}$ & $\mathbf{T}$ & $\mathbf{S}$ & $\mathbf{T}$ & $\mathbf{S}$ & $\mathbf{T}$ & $\mathbf{S}$ & $\mathbf{T}$ & $\mathbf{S}$ \\
\hline Testemunha & 1,0 & 10,0 & 1,0 & 10,0 & 1,0 & 20,0 & 1,0 & 20,0 & 4,0 & 11,1 \\
Enxofre & 1,0 & 0,0 & 1,0 & 0,0 & 1,0 & 0,0 & 1,0 & 0,0 & 4,0 & 0,0 \\
Mancozebe & 1,0 & 10,0 & 1,0 & 10,0 & 1,0 & 40,0 & 1,0 & 30,0 & 4,0 & 50,0 \\
Oxicloreto de Cobre & 1,0 & 26,7 & 1,0 & 16,0 & 1,0 & 20,0 & 1,0 & 20,0 & 4,0 & 0,0 \\
\hline
\end{tabular}

$\mathrm{T}=$ tempo mediano no qual ocorreu mudança de ínstar de $50 \%$ das ninfas.

$\mathrm{S}=$ porcentagem de ninfas que ainda não mudaram de ínstar no tempo $\mathrm{T}$.

O tempo mediano de vida para a fase ninfal de A. gossypii em plantas de pepino sem fungicida (testemunha), foi de quatro dias, e aos seis dias, todas as ninfas já se encontravam na fase adulta. A probabilidade de sobrevivência no tempo mediano de quatro dias foi de 11,1\% (Tabela 1 e Figura $1 \mathrm{~A}$ ).

Quando se empregaram os fungicidas enxofre e oxicloreto de cobre, o tempo mediano de vida da fase ninfal também foi de quatro dias, sem probabilidade de sobrevivência para ambos. Esses resultados evidenciaram que enxofre e oxicloreto de cobre não influenciaram a fase ninfal, enquanto, nos demais tratamentos algumas ninfas ainda permaneceram nessa fase após o tempo mediano de vida (Tabela 1).

Com o fungicida mancozebe, o tempo mediano de vida da fase de ninfa foi de quatro dias, quando foi possível verificar que todas as ninfas sobreviventes mudaram para a fase adulta até o sétimo dia. O total de ninfas que passaram para a fase adulta no tempo mediano foi de 50,0\% (Tabela 1 e Figura 
1A). Kocourek et al. (1994) estudando a biologia de A. gossypii em pepino a $25 \mathrm{oC}$, observaram a duração de 5,0 dias na fase ninfal desse afídeo. $\mathrm{Na}$ mesma temperatura e em dois cultivares de pepino,
Vansteenis e El-Khawass (1995) verificaram duração semelhante para essa fase, sendo de 4,6 dias na cultivar Aramon e de 3,5 dias na cultivar Sporu.

Figura 1. Curvas de sobrevivência para o tempo mediano de vida (dias) para a fase ninfal (A), fase adulta (B) e ciclo biológico (C) de Aphis gossypii mantidos em plantas de pepino tratadas com fungicidas em casa-de-vegetação.
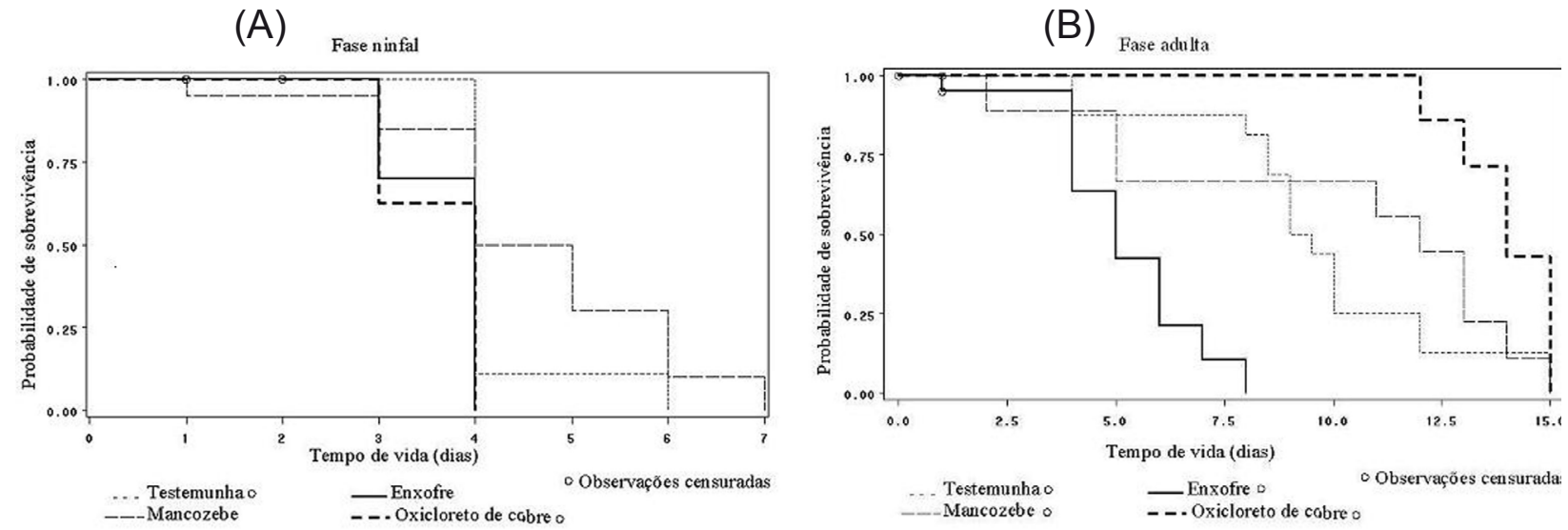

(C) Cielo biologico

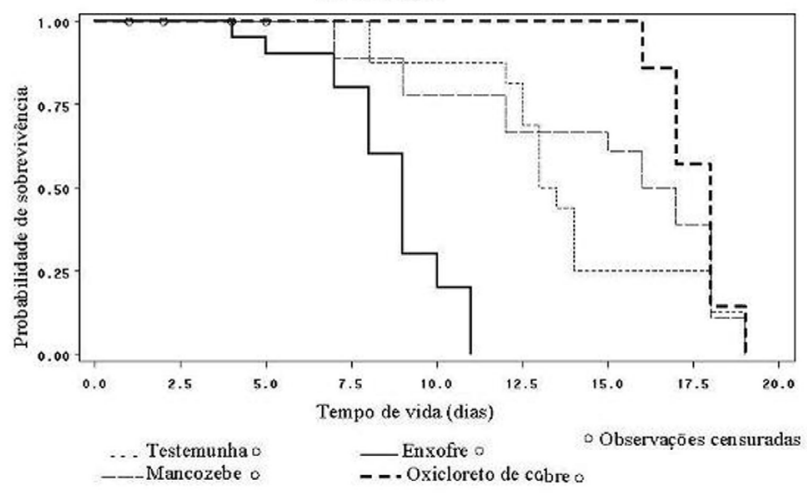

Imagos que não foram submetidos aos fungicidas apresentaram o tempo mediano de vida de 9,3 dias e probabilidade de sobrevivência de $43,8 \%$ (Tabela 2 e Figura 1B).

Para os adultos criados em plantas de pepino que foram tratadas com enxofre, $o$ tempo mediano de vida foi de cinco dias. A probabilidade de sobrevivência, nesse tempo, foi de $42,2 \%$ (Tabela 2) e aos oito dias, todos os adultos já haviam morrido (Figura 1B).
Adultos de A. gossypii em plantas tratadas com mancozebe apresentaram o tempo mediano de vida de 12 dias. $\mathrm{O}$ total de adultos que morreram no tempo mediano foi de $55,6 \%$ (Tabela 2). Para os adultos do tratamento com oxicloreto de cobre, o tempo mediano de vida foi de 14 dias com probabilidade de sobrevivência de $42,9 \%$, ou seja, $57,1 \%$ dos adultos já haviam morrido nesse tempo mediano. 
Tabela 2. Tempo mediano de vida (T) em dias, probabilidade de sobrevivência (S) em \% de adultos e do ciclo biológico total (fase ninfal e fase adulta) de Aphis gossypii mantidos em plantas de pepino tratados com fungicidas em casa-de-vegetação.

\begin{tabular}{lccccc}
\hline Tratamentos & \multicolumn{3}{c}{ Fase Adulta } & & \multicolumn{3}{c}{ Ciclo biológico } \\
\cline { 2 - 3 } Testemunha & $\mathbf{T}$ & $\mathbf{S}$ & & $\mathbf{T}$ & $\mathbf{S}$ \\
Enxofre & 9,3 & 43,8 & 13,3 & 43,8 \\
Mancozebe & 5,0 & 42,2 & 9,00 & 30,0 \\
Oxicloreto de cobre & 12,0 & 44,4 & 16,5 & 50,0 \\
\hline
\end{tabular}

$\mathrm{T}=$ tempo mediano no qual ocorreu morte de $50 \%$ dos adultos (fase adulta) ou morte de $50 \%$ de pulgões durante todo o seu ciclo de desenvolvimento (ciclo biológico). $\mathrm{S}=$ porcentagem de adultos que ainda não morreram no tempo $\mathrm{T}$.

Para os pulgões que não foram submetidos a nenhum tratamento com fungicida, o ciclo biológico apresentou tempo mediano de vida de 13,3 dias, com probabilidade de sobrevivência de 43,8\%. Com enxofre, os pulgões A. gossypii apresentaram, tempo mediano de vida de 9 dias, sendo a probabilidade de sobrevivência, nesse tempo de 30,0\%, e aos 12 dias, todos os adultos desse tratamento já haviam morrido (Tabela 2 e Figura 1C).

Os pulgões criados em plantas de pepino tratadas com mancozebe, tiveram tempo mediano de vida de 16,5 dias. $O$ total de pulgões que morreram no tempo mediano foi de 50,0\%. Com oxicloreto de cobre, o ciclo biológico de $A$. gossypii apresentou tempo mediano de vida de 18 dias, com $85,7 \%$ dos pulgões completando seu ciclo de vida nesse tempo (Tabela 2 e Figura 1C). Vansteenis e El-Khawass (1995) trabalhando com a mesma espécie de afídeo e a $25^{\circ} \mathrm{C}$, observaram que na cultivar Sporu de pepino, a duração do ciclo biológico total foi de 17,8 dias.

O número de ínstares de $A$. gossypii em plantas de pepino não foi influenciado pelos fungicidas testados, sendo constatados quatro ínstares. Soglia, Bueno e Sampaio (2002), Pessoa et al. (2004) e Satar, Kersting e Uygun (2005) obtiveram resultados semelhantes para esse mesmo afídeo em crisântemo, algodoeiro e plantas de pepino.

\section{Conclusões}

Os fungicidas oxicloreto de cobre e mancozebe causaram aumento do tempo mediano de vida na fase adulta e no ciclo biológico total de $A$. gossypii. Já com a aplicação de enxofre o tempo mediano de vida foi reduzido em ambos os parâmetros.

A utilização dos fungicidas oxicloreto de cobre e mancozebe deve ser menos indicada para os produtores de pepino, pois ao controlar as doenças fúngicas haverá aumento do ciclo biológico e da fase adulta do pulgão $A$. gossypii, causando maiores danos devido ao maior tempo sobre o hospedeiro.

\section{Agradecimentos}

Os autores agradecem a Coordenadoria de Aperfeiçoamento de Pessoal de Nível Superior (CAPES) pela bolsa de mestrado concedida ao primeiro autor.

\section{Referências}

AKHTAR, S.; VAN EMDEN, H. F. Effect of the systemic fungicide benomyl on the symbionts and mycetocytes of the bird cherry-oat aphid (Rhopalosiphum padi) (Homoptera: Aphididae) reared on wheat plants. Bulletin of Entomological Research, Wallingford, v. 86, n. 4, p. 319-328, aug. 1996.

. The effect of the systemic fungicide benomyl 
on survival and reproduction of the bird cherry aphid (Rhopalosiphum padi). Annals of Applied Biology, Warwick, v. 120, n. 2, p. 245-255, apr. 1992.

ÁVILA, A. C. de. Viroses de cucurbitáceas. Informe Agropecuário, Belo Horizonte, v. 8, n. 85, p. 52-53, jan. 1982.

BALAZS, K.; MOLNAR, M.; BUJAKI, G.; GONDA, I.; KARACSONY, D.; BARTHA. J. Possibility and problems of organic apple growing in Hungary. Biological Agriculture \& Horticulture, Oxford, v. 15, n. 1/4, p. 223-232, 1997.

BARBOSA, S.; FRANÇA, F. H. Pragas de cucurbitáceas e seu controle. Informe Agropecuário, Belo Horizonte, v. 8, n. 85 , p. 54-56, 1982.

BLACKMAN, R. L.; EASTOP, V. P. Aphids on the word's crops: an identification and information guide, Hoboken: Wiley, 1984. 466 p.

BUENO, V. H. P. Controle biológico em cultivos protegidos: importância e perspectivas. In: SILVA, L. H. C. P. Manejo integrado: doenças e pragas em hortaliças. Lavras: Editora UFLA, 2001. p. 309-331.

COlOSIMO, E. A.; GIOLO, S. R. Análise de sobrevivência aplicada. São Paulo: Ed. Blucher, 2005. $175 \mathrm{p}$.

DEGRANDE, P. E. Guia prático de controle das pragas do algodoeiro. Dourados: UFMS, 1998. $60 \mathrm{p}$.

JULIATTI, F. C. Manejo integrado de fungos fitopatogênicos. In: SILVA, L. H. C. P. Manejo integrado de doenças: doenças e pragas em hortaliças. Lavras: Editora UFLA, 2001. p. 159-220.

KOCOUREK, F.; HAVELKA, J.; BERÁNKOVÁ, J.; JAROSIK, V. Effect of temperature on development rate and intrinsic rate of increase of Aphis gossypii reared in greenhouses cucumbers. Entomologia Experimentalis et Applicata, Dordrecht, v. 71, n. 1, p. 59-64, 1994.

LEITE, M. V. L.; SANTOS, T. M.; SOUZA, B.; CAliXTO, A. M.; CARVAlHO, C. F. Biologia de Aphis gossypii Glover, 1877 (Hemiptera: Aphididae) em abobrinha cultivar Caserta (Cucurbita pepo L.) em diferentes temperaturas. Ciência e Agrotecnologia, Lavras, v. 32, n. 5, p. 1394-1401, 2008.

MATTHEWS, G. A.; TUNSTALL, J. P. Insect pests of cotton. Cambridge: Cab International, 1994. 593 p.

PESSOA, L. G. A.; SOUZA, B.; CARVALHO, C. F.; SILVA, M. G. Aspectos da biologia de Aphis gossypii Glover (1877) (Hemiptera: Aphididae) em quatro cultivares de algodoeiro, em laboratório. Ciência e Agrotecnologia, Lavras, v. 28, n. 6, p. 1235-1239, 2004.
SAS INSTITUTE. SAS/stat: users guide. Cary, NC: SAS Inst, 1990.

SATAR, S.; KERSTING, U.; UYGUN, N. Effect of temperature on development and fecundity of Aphis gossypii Glover (Homoptera: Aphididae) on cucumber. Journal of Pest Science, Heidelberg, v. 78, n. 3, p. 133137, 2005.

SOGLIA, M. C. M.; BUENO, V. H. P.; SAMPAIO, M. V. Desenvolvimento e sobrevivência de Aphis gossypii Glover (Hemiptera, Aphididae) em diferentes temperaturas e cultivares comerciais de crisântemo. Neotropical Entomology, Londrina, v. 31, n. 2, p. 211216, 2002.

VANSTEENIS, M. J.; EL-KHAWASS, K. A. M. H. Life history of Aphis gossypii on cucumber: influence of temperature, host, plant and parasitism. Entomologia Experimentalis et Applicata, Dordrecht, v. 76, n. 2, p. 121-131, 1995.

WEBER, A.; KRANZ, J. Investigations on the influence of fungicides on pests in the agroecosystem winter-wheat. Zeitschrift fur Pflanzenkrankheiten und pflanzenschutz. Journal of Plant Diseases and Protection, Stuttgart, v. 101, n. 2, p. 160-172, apr. 1994. 
